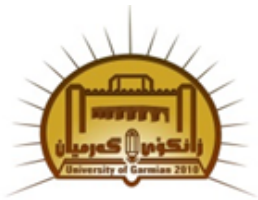

Available online at http://jgu.garmian.edu.krd

Journal of Uni versity of Garmian

https://doi.org/10.24271/garmian.2001

\title{
Evolution of the optical properties of PVA film filled with $\mathrm{CuCl}$
}

\author{
Rezhna Mhammed Kuekha M. \\ Physics Department, College of Education, University of Garmian
}

\section{Article Info}

Received: January, 2019

Revised:February,2019

Accepted:April,2019

\section{Keywords}

Poly vinyl(PVA), casting method, optical constant, optical band gap, Tauc's plot, doping effect, Copper Chloride $(\mathrm{CuCl})$.

\section{Corresponding Author}

rezhna.mhammed@garmian.edu.krd

\begin{abstract}
Polyvinyl alcohol (PVA) has been doped by different percentage of Copper Chloride $(\mathrm{CuCl})$, films for different weight ratio $(0,1,1.5$, and $2 \%)$ were prepared by the well-known solution cast method. The optical properties were obtained in the wavelength range (190-1100) nm. Results show that for the pure PVA films the optical constants were increased after doping and with increasing impurity concentration. For indirect allowed transition the optical energy gap decreases from $4.6 \mathrm{eV}$ (for pure PVA) to $3.8 \mathrm{eV}$ (for $\mathrm{PVA}+2 \% \mathrm{CuCl})$.

The results show the absorption edge for electrolyte samples moves toward a lower energy region by increasing the salt concentration, leading to the band gap reduction. This study shows that the optical properties of PVA are affected by salt concentration.
\end{abstract}

\section{Introduction}

The mixing of filler and functional polymers (polar polymers) interacting at atomic level constitute the bases for preparing an important group of inorganics-organic nanostructured materials i.e., polymer nano-composites with a good mechanical strength [D.K. Pradhan et al., 2008]. In recent years there has been an increased attention in the developing of polymers to replace more and more metals [S. Prasher et al., 2014]. Due to the wide applications of optical materials in glass lens, camera lens, optical waveguides, optical reflectors, etc., polymer composites with high-refractive index (RI) have been widely studied. The advantages of organic materials are light weight, their transparency, ease of process, cost-efficiency and good mechanical properties. But they normally show low refractive index [J. Jin at al., 2013]. (Polyvinyl alcohol) (PVA) is a polymeric material which is soluble in water, a good charge storage capacity, has a high dielectric strength, and interesting optical properties [D.M. Fernandes et al., 2013]. To get an important information about the transmittance, absorbance, and reflectance of the observed polymeric films the optical study is very interesting [R. Seoudi 
and A.M.A. Nada 2007]. PVA has carbon chain backbone with hydroxyl groups attached that can be a source of hydrogen bonding which help the formation of polymer nano-composite. Due to its high transparency and ability to form a barrier to oxygen this polymer is a good candidate for incorporation into multilayer coatings of organiczsolar cells [D.M. Fernandes et al., 2013]. These are influenced not only by the structure and nature of the dopant but also by preparation methods and the doping concentration [A. ElKhodary et al., 2008]. In order to modify and improve its properties different additives are usually added to polymer. The optical and electrical properties of PVA (polyvinyl alcohol) highly affected by polymer Inorganic additives such as transition metal salts [Abdul-Aziz M 2011].

The present work was devoted to investigate the effect of $\mathrm{CuCl}$ on some characteristics of polyvinyl alcohol (PVA) films, including dialectical properties and the optical band gap energy, in the UV/VIS region to understanding optical properties which is relatively affected by processing conditions and the mechanism of transitions. In particular, the introducing of inorganic salt into the polymer can improve and modify its optical properties, due to a strong interface interaction between an inorganic salt and the organic polymer.

\section{Solution Cast Method}

Polymer electrolyte films are generally obtained by simple casting procedure. The polymer is the host and the inorganic salts are dissolved in adequate reciprocal compositions in suitable solvents (e.g. acetonitrile, methanol, ethanol, double distilled water, etc.). Appropriate amounts of the salt mixture of the chosen stoichiometry and PEO were separately dissolved in methanol, and the two solutions were then stirred together for approximately 24 hrs. The solutions were prepared in such a way that a ratio of $1 \mathrm{gm}$ of PEO to $100 \mathrm{~cm} 3$ of solvent was maintained, and this was found to be optimum for casting purposes. Solvent was allowed for evaporation at room temperature [Scrosati. B. 1988]. The thickness of the cast film was approximately 100 to $200 \mu \mathrm{m}$. The schematic illustration of the preparation procedure of polymer electrolyte film is shown in Fig.1. [See Fig-1]

This paper is prearranged as follows: following the introduction in Section 1, experimental procedure is presented in Section 2. Section 3 is devoted to results and discussion, and Section 4 includes conclusion.

\section{Experimental procedure}

Polyvinyl alcohol (PVA) polymer with molecular weight $(10000 \mathrm{~g} / \mathrm{mole})$, supplied by (BDH chemicals, England) with high purity (99.999\%) were used as matrix polymeric materials in this work. The films were prepared using the casting method by dissolving the powders with the appropriate percentages in distilled water at $90^{\circ} \mathrm{C}$, and the solution is stirred well using a magnetic stirrer for $1 \mathrm{~h}$ to obtain a homogenous solution.

Copper chloride $(\mathrm{CuCl})$ solution was prepared by dissolving the salt in redistilled water. $(0 \%, 1 \%$, $1.5 \%$, and $2 \%$ ) concentrated of Copper chloride were mixed with PVA matrix. The solution was poured into flat glass plate dishes $(5 \mathrm{~cm}$ diameter). And allowed to evaporate the solvent slowly at room temperature for 2 weeks. The thickness of the films was in the range of $(30 \pm$ 0.05) $\mu \mathrm{m}$ by using micrometer. The transmittance and absorbance measurements were carried out using a Shimadzu UV/VIS$160 \mathrm{~A}$ double beam spectrophotometer in the wavelength range (190-1100) $\mathrm{nm}$.

\section{Result and Discussion}

The first feature that changed after drying the $\mathrm{CuCl}$ doped samples was the color of the sample. The PVA polymer film $(0 \%)$ is colorless and transparent for visible light. The color of the $\mathrm{PVA} / \mathrm{CuCl}$ electrolyte films changed progressively towards light green for $(1 \%, 1.5 \%$, 
and $2 \%$ ) $\mathrm{CuCl}$. These modifications have caused the observed change in the UV-Vis spectroscopy. The Ultraviolet (UV) absorption mainly corresponds to the electron transition from the top of the valence band to the bottom of the conduction band. Optical absorbance measurements were taken for CuCl-Polyvinyl alcohol samples. The optical properties of the films by means of the optical absorption in the $\mathrm{UV}-\mathrm{V}$ is region of (190-1100) $\mathrm{nm}$ have been investigated.

Absorbance as a function of wavelength for the pure PVA and PVA doped samples are shown in Fig. 2. According to the obtained results, it can be seen clearly that the absorption decreases rapidly with increasing wavelength up to 400 $\mathrm{nm}$, one peak has been observed at nearly (250310) $\mathrm{nm}$ in $\mathrm{CuCl}$ doped samples, As indicated in figure that $\mathrm{CuCl}$ salt enhances the absorbance of the PVA host. [See Fig-2]

Transmittance as a function of wavelength for pure PVA polymer film and PVA doped samples were recorded at room temperature and are shown in Fig. 3. Pure PVA is a colorless polymer without any noticeable absorption in the visible range. The figure shows that the transmittance decreases with the increase of the weight percentage of the added $\mathrm{CuCl}$ salt. [See Fig-3]

Absorption is expressed in terms of the absorption coefficient $(v)$, which is defined as the relative decrease rate in light intensity. $(v)$ (cm)-1 can be calculated from the optical absorption spectra $A$ using the Beer Lambert's formula [S. Elliot 1998; O. Gh. Abdullah et al., 2015]:

$\alpha(v)=\frac{1}{d} \log \left(\frac{I^{\circ}}{I}\right)=\frac{2.303}{d} A$

where $d$ is the sample thickness; $I_{\mathrm{o}}$ and $I$ are the incident and transmitted intensities, respectively [A.A. Jamous and A.M. Zihlif 2010]. At high energies, absorption coefficient is high. This means that there is a high possibility for electron transition. Consequently, the energy of incident photon is enough to move the electron from the valence band to the conduction band, i.e. the energy of the incident photon is greater than the energy gap [El-Dahshan M. E. 2002]. We can also see from Fig. 4 that the absorption coefficient increases with increasing the weight percentage of the added $\mathrm{CuCl}$ salt. [See Fig-4]

Fig.5 presents the optical absorption coefficients $(\alpha)$ for un-doped and $\mathrm{CuCl}$ doped PVA films versus photon energies. The absorption coefficient increases with increasing the photon energy (hv) as well as $\mathrm{CuCl}$ concentration. [See Fig-5]

We can obtain the refractive index (n) as a function of wavelength from

$n=\left(\frac{1+R}{1-R}\right)+\sqrt{\frac{4 R}{(1-R)^{2}}-k^{2}}$

where $R$ : reflectance, and $k$ : the extinction coefficient, which is related to the absorption coefficient $\alpha(v)$ and wavelength $\lambda$ by [F. Yakuphanoglu and H. Erten 2005]: in which $k=$ $\lambda \alpha / 4 \pi$

Fig. 5 and 6 show the photon energy dependence of refractive index and the extinction coefficient for pure PVA and $\mathrm{CuCl}$ doped PVA films. It can be discerned from Fig. 6 that the refractive index of $\mathrm{CuCl}$ doped PVA films is lower than the refractive index (n) of pure PVA and it decreases with increasing concentration of $\mathrm{CuCl}$ in PVA matrix. [See Fig-6]

The extinction coefficient $(\mathrm{k})$ increases with the increasing of photon energy for all samples and with increasing impurity concentration as in Fig. 7. The extinction coefficient $k$ of the doped samples have a peak at $E=4.75 \mathrm{eV}$, which increases with increasing concentration of $\mathrm{CuCl}$ in PVA dielectric medium. [See Fig-7]

Reflectance $(\mathrm{R})$ of the films could be calculated using the following relation [Zahr El-Deen et al., 2014]:-

$\mathrm{R}+\mathrm{T}+\mathrm{A}=1$

Where: T represent is the transmittance. Plot of reflectance $R$ against photon energy are displayed in Fig. (8). It is clear from the figure 
that the reflectance of the pure PVA film increases gradually with increasing of photon energy within the range (3.15- 5.2) $\mathrm{eV}$, this increasing indicate that the absorption is very low in region under energy gap, and decreasing the transmittance in this range by the equation (3). After doping the reflectance for all samples is increased in low photon energies region and moving the curves vertex for direction this energy, then it decreased in high energies before and after doping due to a decreasing in the energy gap with increasing impurity percentage [Zahr El-Deen et al., 2014]. The reflectance is increases with increasing impurity percentage. New peaks were appearing in energies (4.2) eV for sample (1\%), (4.1) $\mathrm{eV}$ and (3.95) $\mathrm{eV}$ for samples $(1.5 \%$ and $2 \%)$, which is an indication of change in the molecular structure degradation, polymer fragments, or free radicals of PVA and/or PVA/CuCl films [J. Tauc 1974]. [See Fig-8].

The complex dielectric function is $\varepsilon=\varepsilon r+\varepsilon i$ where $\varepsilon \mathrm{r}$ is the real part and $\varepsilon \mathrm{i}$ is the imaginary part of dielectric constant. The real part and imaginary part of dielectric constant are expressed as

$\varepsilon_{r}=n^{2}-k^{2}$,

$\varepsilon_{i}=2 n k$

It is clear from this figure that the imaginary part depends on extinction coefficient according to equation (5) because the refractive index value is very small. The real and imaginary parts dependences on photon energy of samples are shown in Fig. 9 and 10, respectively. It can be concluded that $\varepsilon r$ is larger than $\varepsilon$ i because it mainly depends on $\mathrm{n} 2$. After doping the real part was increased with increasing impurity concentration. [See Fig-9]

After doping with $\mathrm{CuCl}$ the imaginary part of dielectric constant for the samples was increased with the increasing of photon energy and with increasing the doping concentration. [See Fig-10]
According to the Taucs relation [O.G. Abdullah et al. 2013], the absorption coefficient $\alpha$ of band gap material is given by:

$\alpha h v=\beta\left(h v-E_{g}\right)^{\gamma}$

where $E \mathrm{~g}$ is the energy band gap, constant $\beta$ is different for different transitions, and is also a useful diagnostic of the material. $h v$ : the energy of photons, and $\gamma$ : an index used to be assumed the values $2,3,1 / 2$ or $3 / 2$, corresponding to allowed indirect and forbidden indirect, allowed direct, forbidden direct transitions, respectively depending on the nature of the electronic transition, [C. F. K. Lingshirn 1997].

The indirect band gap values were obtained from the plot of $(\alpha h v) 1 / 2$ versus $h v$. The extrapolation of the straight line to $(\alpha h v) 1 / 2=0$ gives value of the indirect band gap of the material, as shown in Fig. 11. All these values of indirect band gap values are listed in Table 1 . [See Fig-11]

The direct band gap values were obtained from the plot of $(\alpha h v) 2$ versus $h v$. The extrapolation of the straight line to $(\alpha h v) 2=0$ gives value of the direct band gap of the material, as shown in Fig.12. All these values of direct band gap values are listed in Table 1. [See Fig-12]

Absorption coefficient can be expressed according to Urbach relation [El-Bashir and S. M. 2012]:

$\alpha=\alpha_{0} \exp \left(\frac{h v}{E_{u}}\right)$

Where $\alpha_{0}$ is constant and Eu: is Urbach energy for localized states. The Urbach energy can be calculated from the inverse of the slope of $(\ln \alpha)$ versus (hv). One can observe from the Fig.13 that the Urbach energy values were decreased with the decreasing of energy gap and with the increasing of impurity concentrations, this behavior because the increase of defect levels in the allowed band gap by increasing $\mathrm{CuCl}$ content [Pankove, J. I. 1975]. All these values of the 
Urbach energy values are listed in Table 1. [See Fig-13]

Table 1: Values of indirect band gap and direct band gap energies for pure and doped PVA films.

\begin{tabular}{lccc} 
Samples & $\begin{array}{c}\text { Indirect } \\
\text { Energy Gaps } \\
\text { (Ev) }\end{array}$ & $\begin{array}{c}\text { Direct } \\
\text { Energy } \\
\text { Gaps (Ev) }\end{array}$ & $\begin{array}{c}\text { Urbach } \\
\text { Energy } \\
\text { (Ev) }\end{array}$ \\
\hline $0 \%$ CUCL & 4.6 & 4.4 & 0.186 \\
$1 \%$ CUCL & 4.1 & 2.9 & 0.149 \\
$1.5 \%$ & 3.9 & 2.8 & 0.112 \\
CUCL & 3.8 & 2.7 & 0.107 \\
$2 \%$ CUCL & & & \\
\hline
\end{tabular}

The optical conductivity was obtained using the relation:

$\sigma=\frac{\alpha n c}{4 \pi}$

Where $\sigma$ represent the optical conductivity, $\mathrm{c}$ is the velocity of light. Figure 14 shows the variation of optical conductivity $(\sigma)$ as a function of wavelength. It is clear that the optical conductivity increases with increasing concentration of $\mathrm{CuCl}$. [See Fig-14]

\section{Conclusion}

In this work, the effect of $\mathrm{CuCl}$ impurity on the optical properties of PVA films has been studied, using solution cast technique. The absorbance and the absorption coefficient for (PVA-CuCl) films increases with increasing of the filler content (wt. \%). In general energy band gap (Eg) decreases with doping. The doping process decreases the transmittance. The type of electronic transition was indirect allowed transition. while refractive index, extinction coefficient and real and imaginary parts of dielectric constant increase with increasing of filler content (wt. \%). The optical conductivity increase with increasing concentration of $\mathrm{CuCl}$ is attributed to an increase of charge carrier concentration.

\section{References}

1- El-Khodary, A.H. Oraby, and A.E. Youssef,"The effect of AgNO3 filler on the physical properties of polystyrene films", International J. of Mat. Sci. 3(1), 11-24 (2008).

2- A.A. Jamous, A.M. Zihlif, "Study of the electrical conduction in poly (ethylene oxide) doped with iodine", Physica B: Condensed Matter 405, 2762-2767 (2010).

3- A.N. Ananth, S. Umapathy, J. Sophia, T. Mathavan, D. Man- galaraj, On the optical and thermal properties of in situ/ex situ reduced Ag NP's/PVA composites and its role as a simple SPR- based protein sensor. Appl. Nanosci. 1, 87-96 (2011).

4- Abdul-Aziz M.; "Cerium (III) doping effects on optical and thermal properties of PVA films"; Physica B: Condensed Matter. 406(6-7), p.1300 (2011).

5- Uma Devi, A.K. Sharma, and V.V.R.N. Rao, "Electrical and optical properties of pure and silver nitrate-doped polyvinyl alcohol films", Material Letters 56, 167-174 (2002).

6- K. Lingshirn, "Semiconductor Optics", Springer Verlag, Berlin Hridelberg, (1st ed.), (1997).

7- D.K. Pradhan, R.N.P. Choudhary, B.K. Samantaray, Studies of structural, thermal and electrical behavior of polymer nano composite electrolytes. Exp. Polym. Lett. 2, 630-638 (2008).

8- D.M. Fernandes, J.L. Andrade, M.K. Lima, M.F. Silva, L.H.C. Andrade, S.M. Lima, A.A.W. Hechenleitner, E.A.G. Pineda, Thermal and photochemical effects on the structure, morphology, thermal and optical properties of PVA/Ni0.04Zn0.96O and PVA/ $\mathrm{Fe} 0.03 \mathrm{Zn} 0.97 \mathrm{O}$ nano composite films. Polym. Degrad. Stab. 98, 1862-1868 (2013).

9- El-Bashir, S. M.; "photophysical properties of florenscent PMMA/SiO2 nano hybrids for solar energy appl-ications"; Journal of Luminescence. 132, 1786-1791, 2012. 
10- El-Dahshan M. E., "Introduction to Material Science and Engineering", 2nd Ed., King Saud University Press, Saudi Arabia (2002).

11- F. Yakuphanoglu, H. Erten, "Refractive index dispersion and analysis of the optical constants of an ionomer thin film", Optical Applicate 35, 969-976 (2005).

12- J. Tauc, Plenum Press, in Amorphous and liquid semiconductor, New York, p. 159 (1974).

13- J. Jin, R. Qi, Y. Su, M. Tong, J. Zhu, Preparation of high refractive-index PMMA/TiO2 nano composites by one-step in situ solvothermal method. Iran. Polym. J. 22, 767-774 (2013).

14- O.G. Abdullah, D.A. Tahir, S.S. Ahmad, H.T. Ahmad, IOSR Journal of Applied Physics, 4, 52-57 (2013).

15- O.Gh. Abdullah, D.R. Saber, L.O. Hamasalih, "Complexion formation in PVA/PEO/CuC12 solid polymer electrolyte", Universal Journal of Materials Science, 3, 15 (2015).

16- Pankove, J. I.; "Optical processes in semiconductors", Dover Publications, Inc. New York, 91; (1975).
17- Seoudi, A.M.A. Nada, Molecular structure and dielectric properties studies of chitin and it's treated by acid, base and hypochlorite. Carbohydr. Polym. 68, 728-733 (2007).

18- Elliot, The Physics and Chemistry of Solids, John Wiley \& Sons, New York, (1998).

19- Prasher, M. Kumar, S. Singh, Electrical and optical properties of O6+ ion beam-irradiated polymers. Int. J. Polym. Anal. Charact. 19, 204-211 (2014).

20- Scrosati.B., solid State Ionic Devices, Eds. Chowdari, B.V.R. and Radhakrishna.S., World Scientific Publisng Co. 133 (1988).

21- Raja, A.K. Sarma, and V.V.R. Narasimha Rao, "Optical properties of pure and doped PMMA- COP4VPNOPolymer films", Materials Letters, 57 (30), 4678-4683 (2003).

22- Zahr El-Deen, H.; Hafez, A.I.; "Physicschemical stability of PVA films doped with $\mathrm{Mn}+2$ ions against weathering conditions"; The Arabian Journal for science and engineering. 3. (0A), 03-26, (2014). 


\section{Figures}

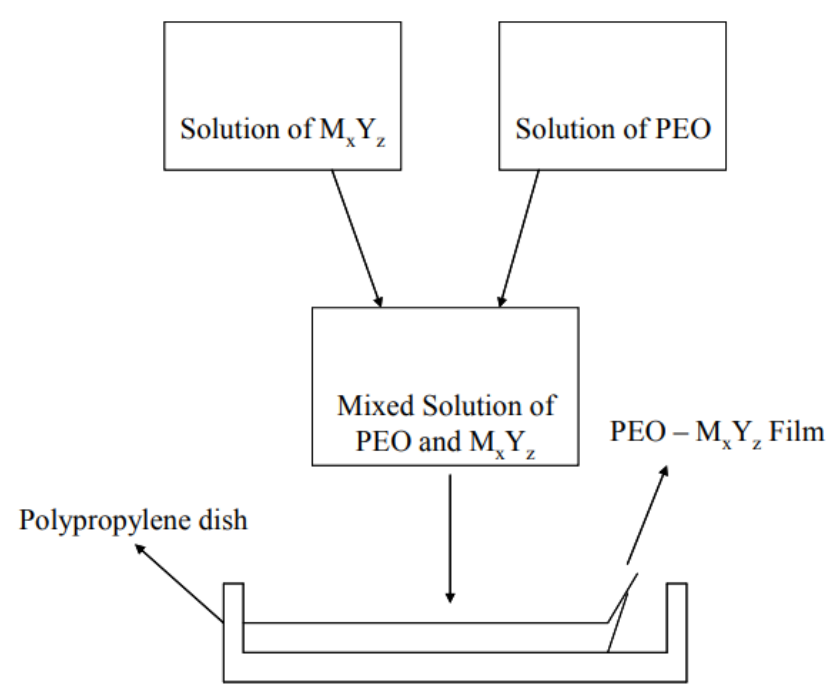

Fig.1: Schematic illustration of the preparation procedure of polymer electrolyte film by solution casting method

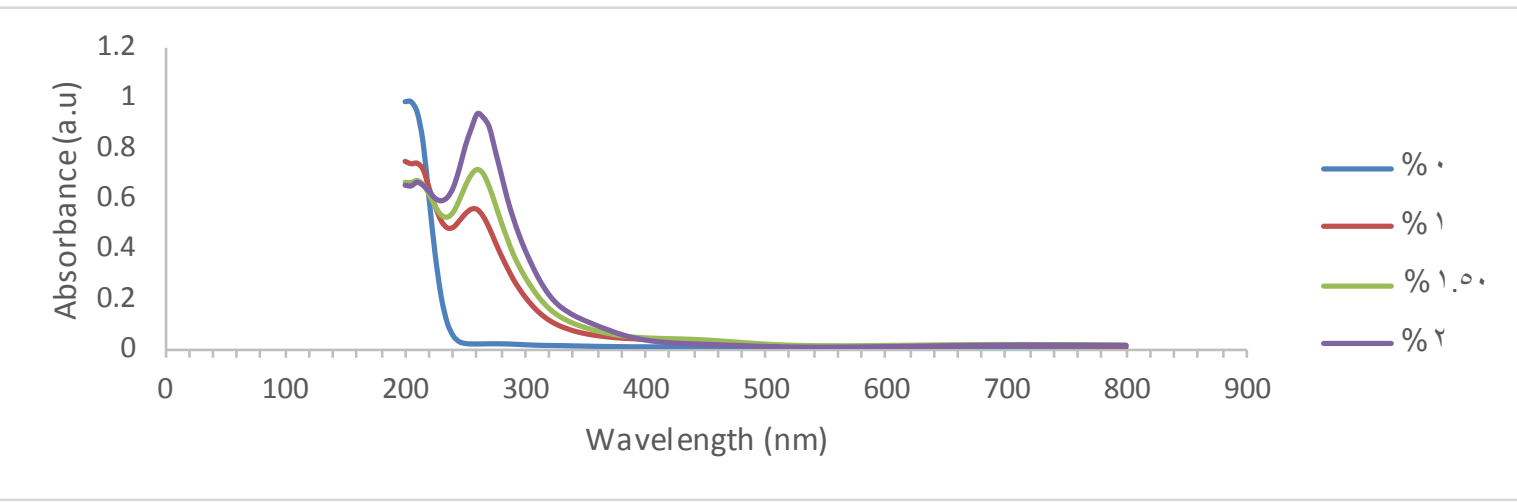

Fig-2 Variation of the Optical absorption (a.u) Vs Wavelength (nm) for pure and doped PVA at room temperature

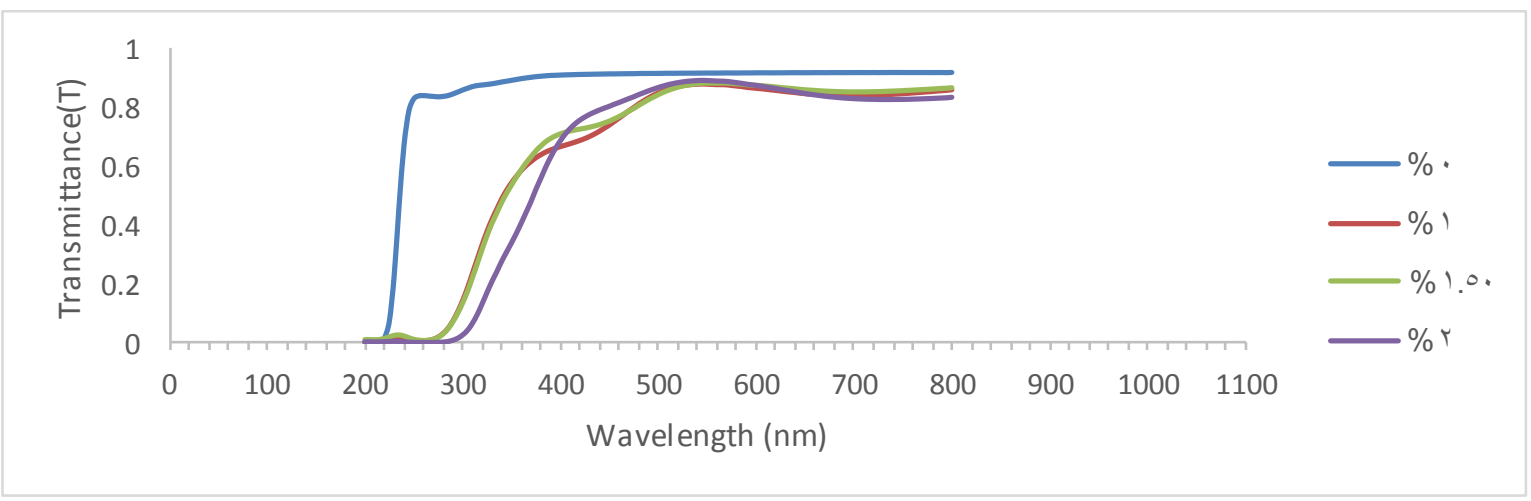

Fig. 3 Transmittance Spectra for (PVA-CuCl) with different $\mathrm{CuCl}$ concentration as a function of wavelength 


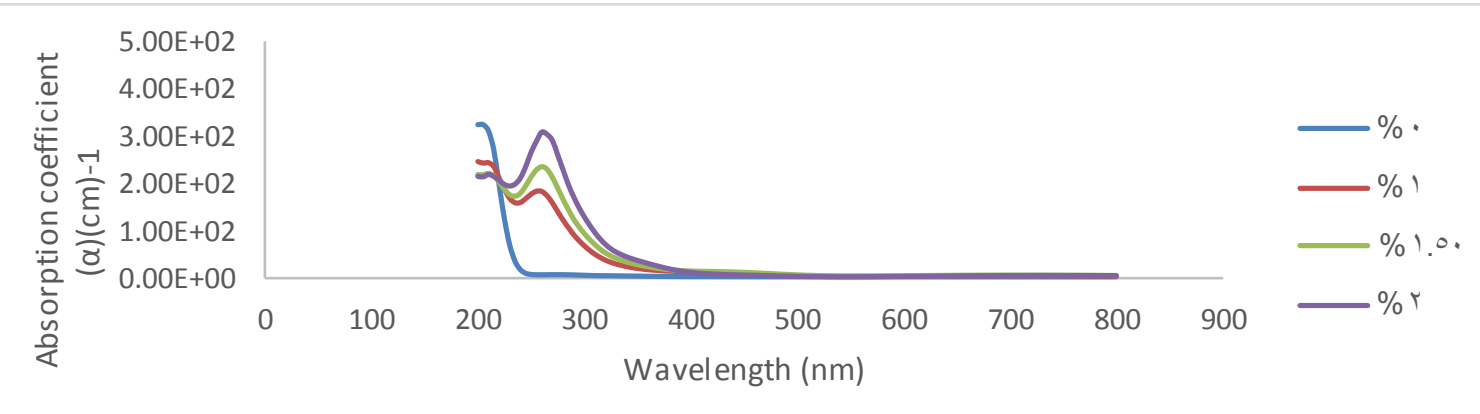

Fig. 4 Absorption Coefficient $\alpha(\mathrm{cm})-1$ for (PVA-CuCl) with different $\mathrm{CuCl}$ concentration as a function of wavelength $(\mathrm{nm})$

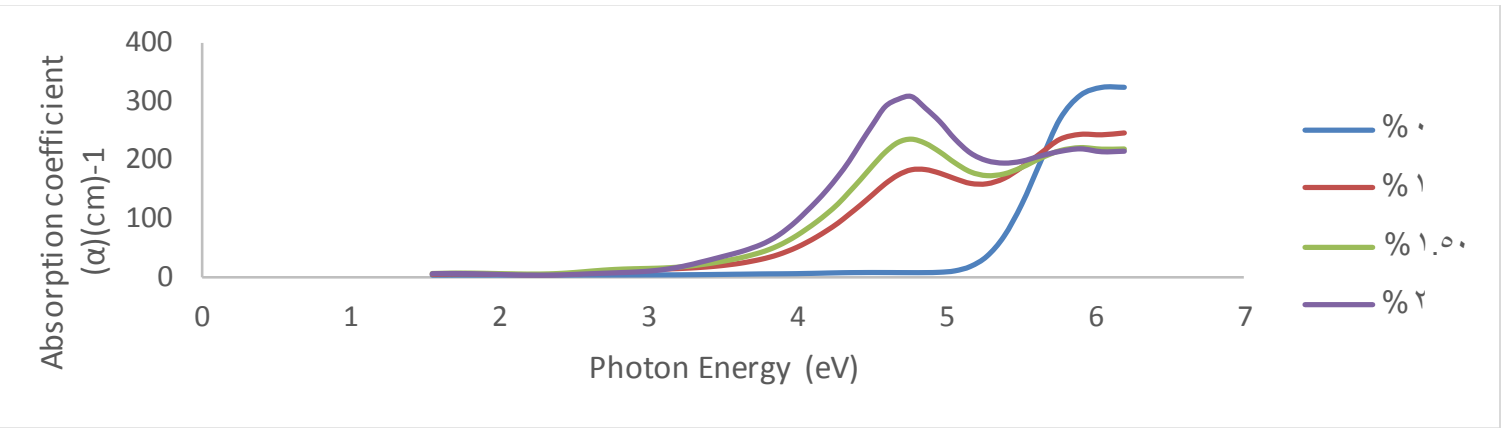

Fig. 5 Absorption Coefficient $\alpha(\mathrm{cm})-1$ for (PVA-CuCl) with different $\mathrm{CuCl}$ concentration as a function of Photon Energy (eV)

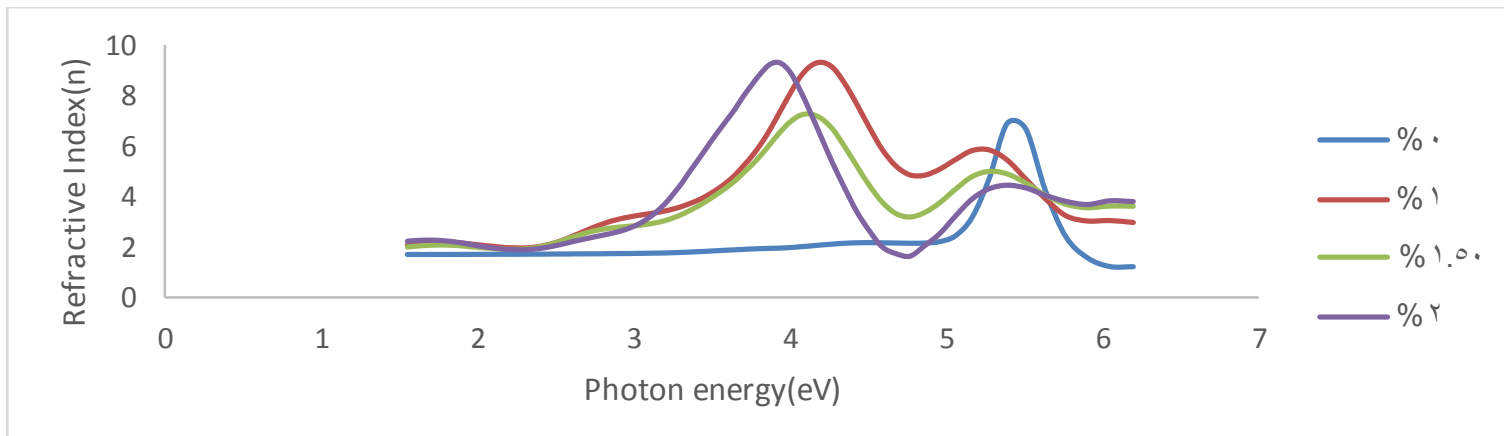

Fig. 6 refractive index (n) for (PVA-CuCl) with different $\mathrm{CuCl}$ concentration as a function of Photon Energy (eV) 


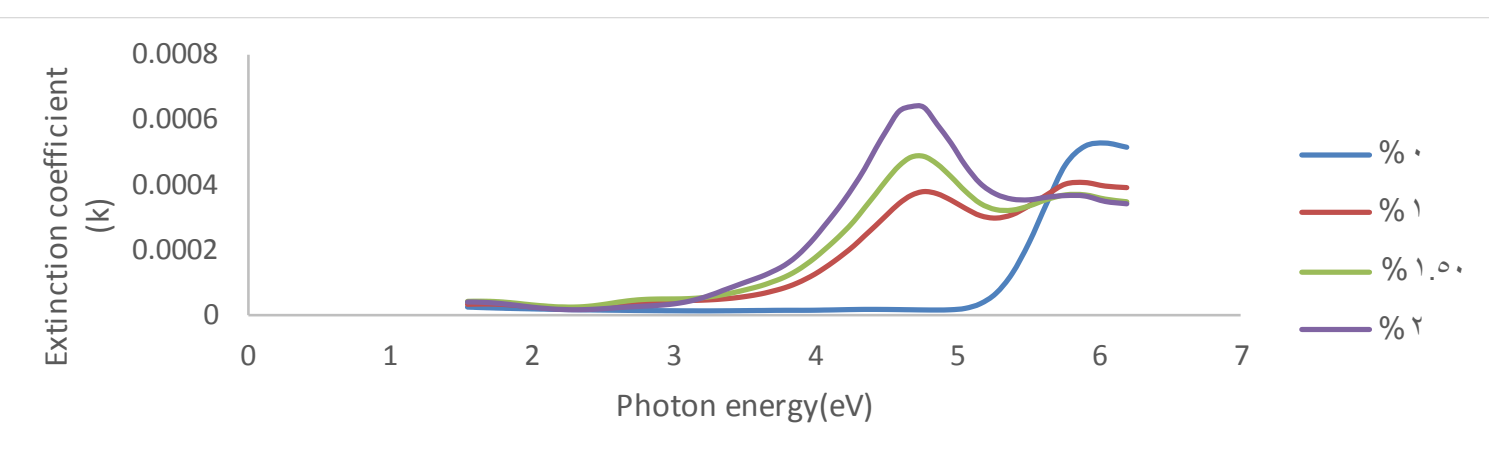

Fig. 7 The extinction coefficient (k) for (PVA-CuCl) with different $\mathrm{CuCl}$ concentration as a function of Photon Energy (eV)

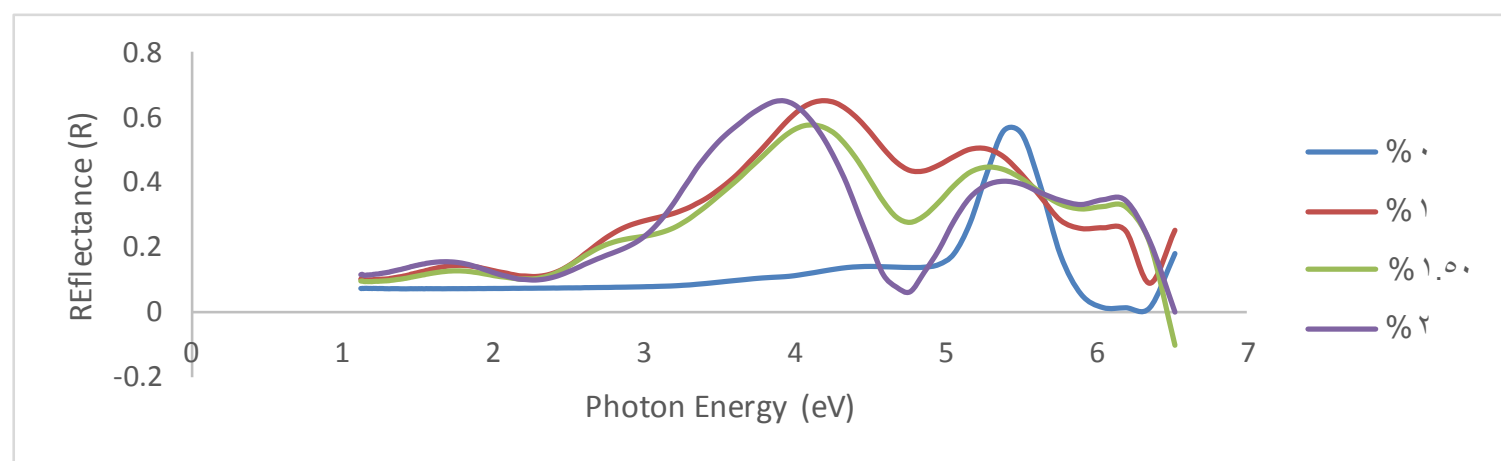

Fig. 8 The Reflectance (R) for (PVA-CuCl) with different $\mathrm{CuCl}$ concentration as a function of Photon Energy $(\mathrm{eV})$

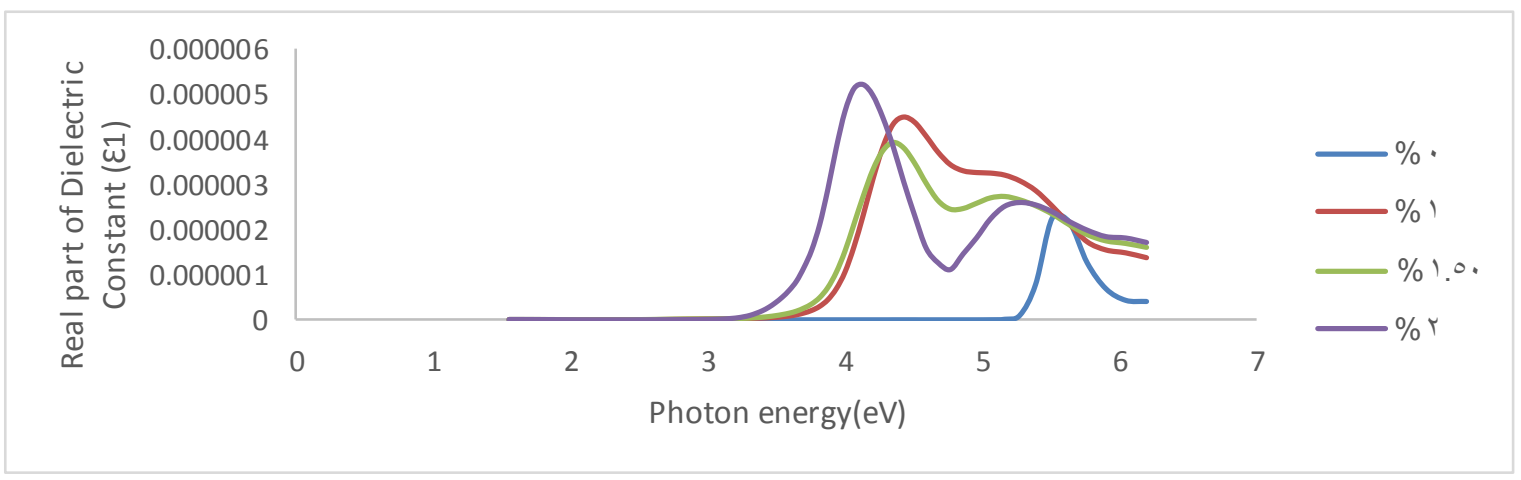

Fig. 9 The real part of dielectric constant for (PVA-CuCl) with different $\mathrm{CuCl}$ concentration as a function of Photon Energy (eV) 


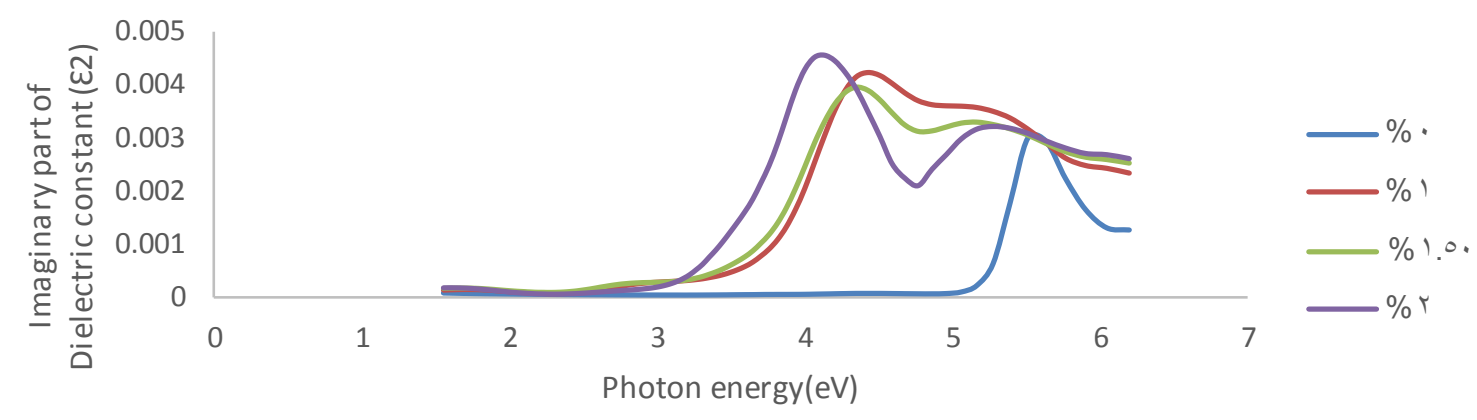

Fig. 10 The imaginary part of dielectric constant for (PVA-CuCl) with different $\mathrm{CuCl}$ concentration as a function of Photon Energy (eV)

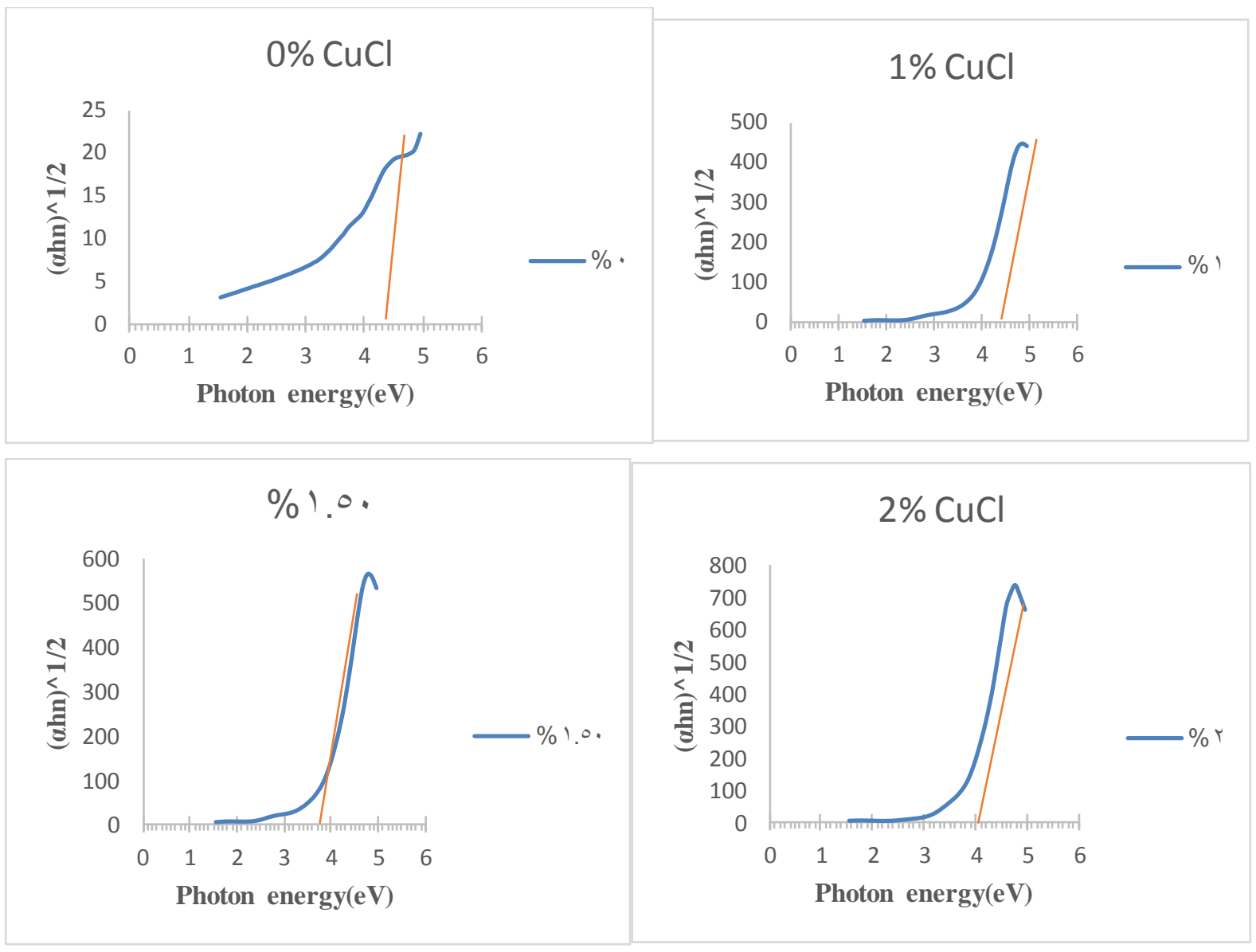

Fig. $11(\alpha h v) 1 / 2$ for (PVA-CuCl) with different $\mathrm{CuCl}$ concentration as a function of Photon Energy (eV) 


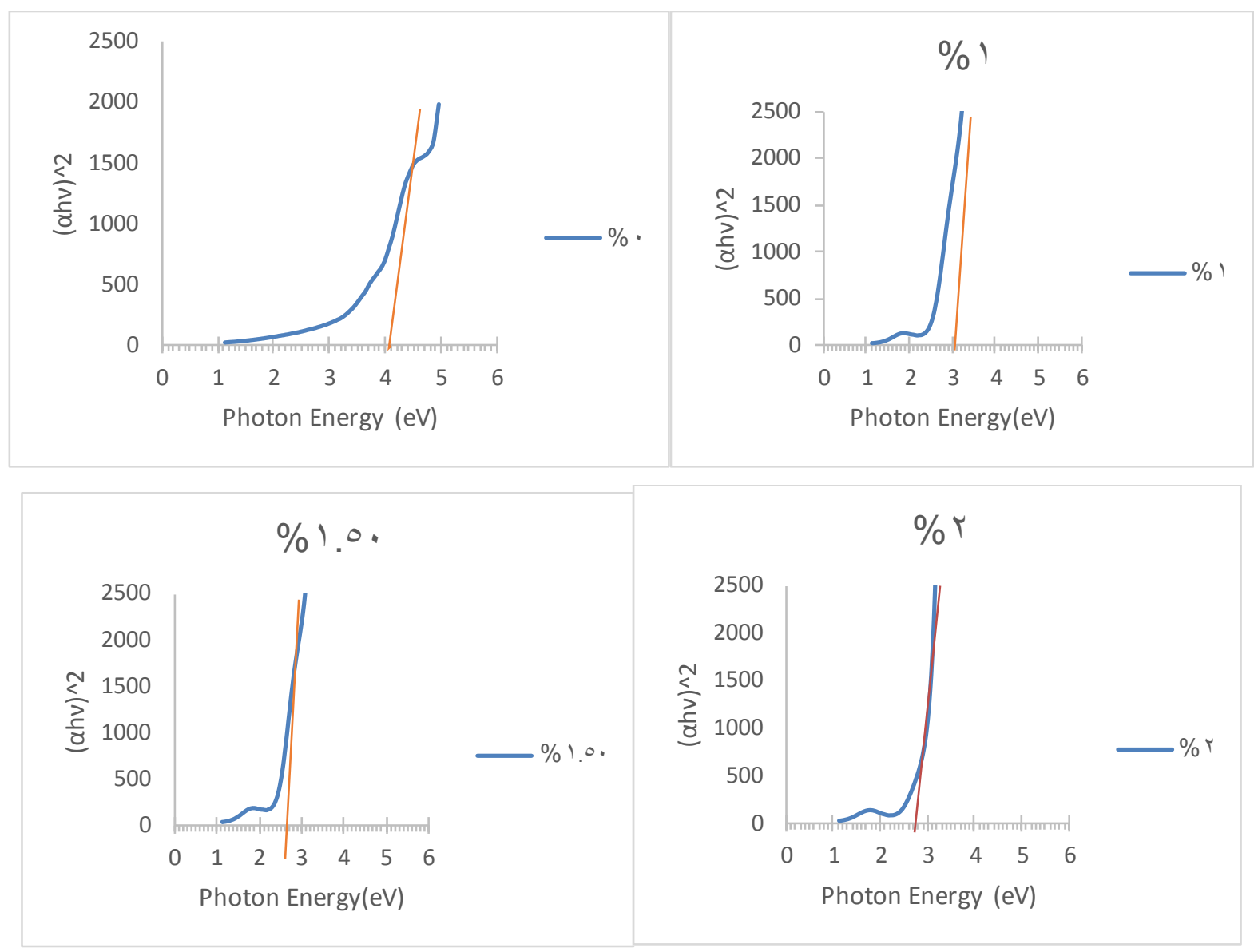

Fig.12 $(\alpha h v) 2$ for (PVA-CuCl) with different $\mathrm{CuCl}$ concentration as a function of Photon Energy (eV)

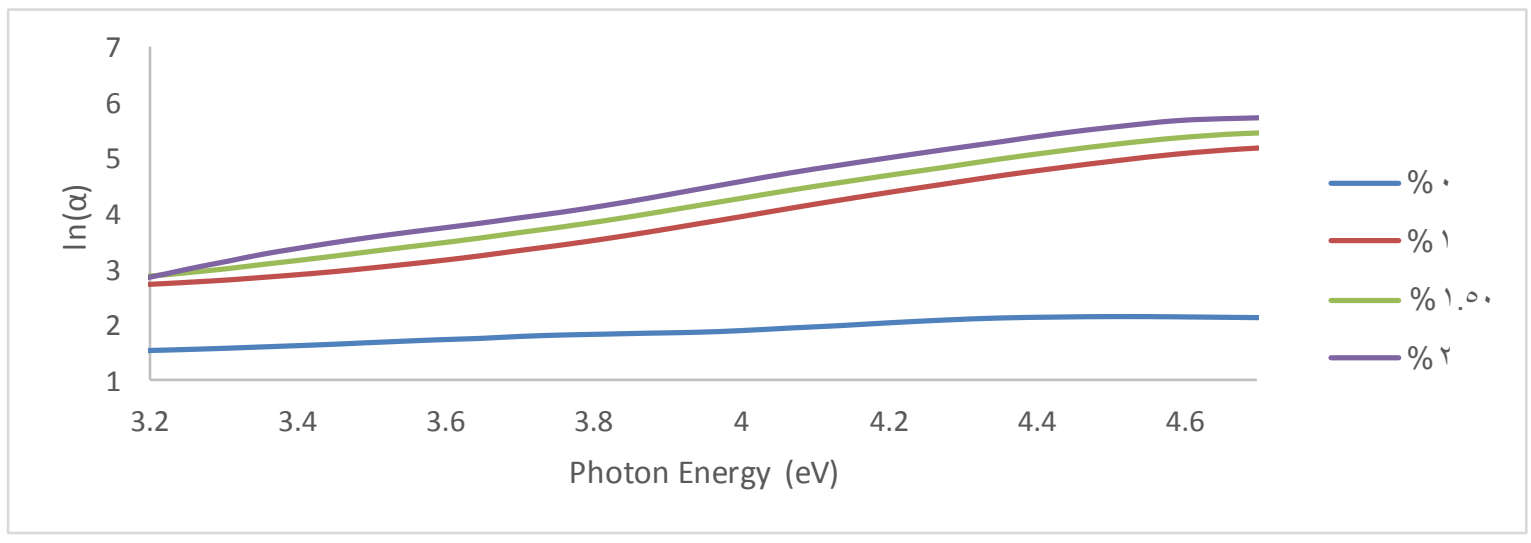

Fig.13 $\ln (\alpha)$ for (PVA-CuCl) with different $\mathrm{CuCl}$ concentration as a function of Photon Energy (eV) 


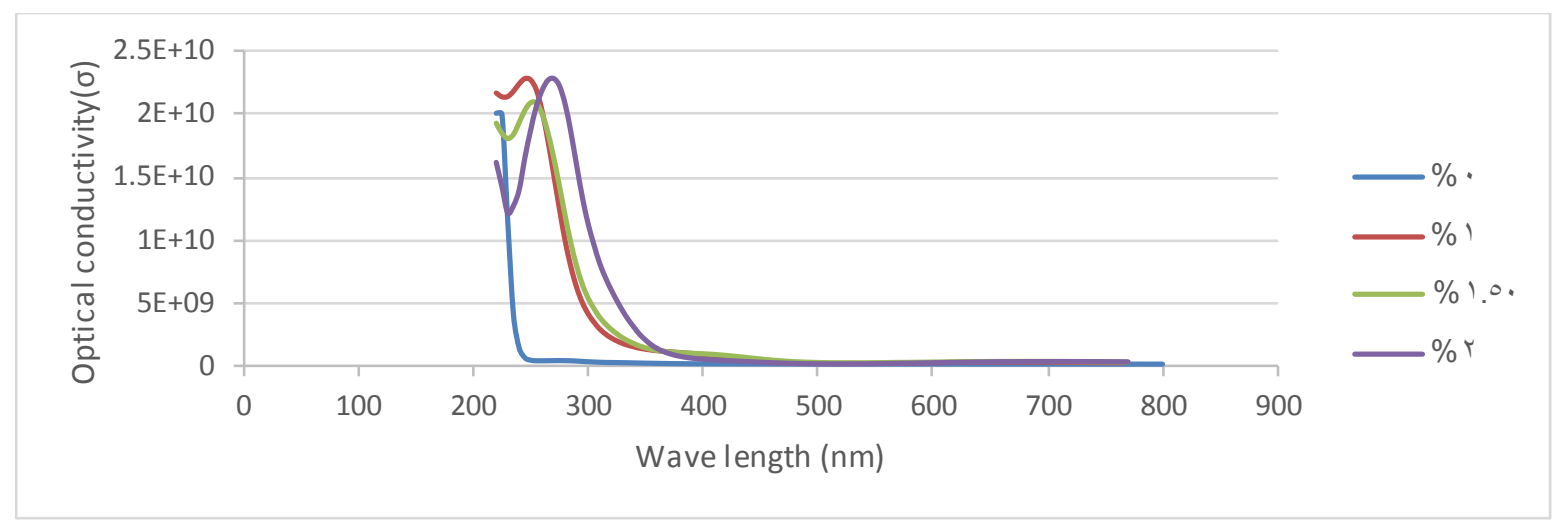

Fig.14 The optical conductivity $(\sigma)$ for (PVA-CuCl) with different $\mathrm{CuCl}$ concentration as a function of wavelength (nm). 